\title{
Hygiene and Dietary Measures in the Treatment of Type 2 Diabetes Population of Chief Region (Algeria)
}

\author{
Ghouini A and Boukoufa $\mathrm{H}^{*}$ \\ Department of Physiology, CHU Blida - University of Blida, Algeria
}

Received: 盋April 16, 2018; Published: 制 April 26, 2018

*Corresponding author: Boukoufa H, Department of Physiology, Faculty of Medicine, CHU Blida - University of Blida, Algeria

\begin{abstract}
Diabetes mellitus is a chronic, sometimes silent disease that exposes cardio-vascular morbidities by its micro and macroangiopathic complications with heavy socio-economic repercussions. Strategies of various kinds are put in place to contain this metabolic and nutritional pathology; hygiene and dietary measures participate in this program to fight against the outbreak and worsening of the diabetic disease.

We wanted in this study to make an inventory of places concerning the therapeutic education, the knowledge of the patients on the disease and the hygienic-dietary rules which should accompany any therapeutic scheme for an optimal control of the evolution of the diabetes. The socio-economic and school level, the food beliefs and the non-access to a specialized consultation, translate the non-observance of the hygienic-dietary measures which cause difficulties optimal control of diabetes.
\end{abstract}

Keywords: Hygienic; Dietary measures; Therapeutic education; Diet; Diabetes

\section{Introduction}

Diabetes mellitus is a chronic, sometimes silent disease that exposes cardiovascular morbidities by its micro and macroangiopathic complications, and other psychological, oncological, cognitive and even arthropathic constraints with adverse socioeconomic consequences. Diabetes is therefore a real public health problem nationally and internationally. All learned societies: American Diabetes Association (ADA), International Diabetes Federation (IDF), Diabetes Control and Complications Trial (DCCT) and even SAD (Algerian Society for Diabetology) have alarm and have put in place many strategies to deal with this problem. The question posed is that of our positioning in relation to the directives of these learned societies concerning:

a) The individualisation of the care of diabetics

b) The involvement of diabetics in this care

c) The adherence of these patients to the different councils of their doctors
Admittedly, the majority of our diabetics, especially those of type 2, are of a low level of education, even illiterate, but the involvement and adherence of these people to the orientations of care is still possible and very useful. This modest study contributes part of the answer to this problem concerning the current state of diabetic patients in a region of the Wilaya de Chlef (west central Algeria) in terms of information (therapeutic education), knowledge about disease and the rules of hygiene and dietary (RHD) to accompany any therapeutic scheme. In order to better understand the relationship of diabetic subjects with medical care, we were led to introduce into the interview questions focused on dietary and dietary rules and to provide an overview of patients' knowledge. diabetics on:

a) The meaning of lifestyle and dietary measures;

b) Their knowledge about RHD;

c) Diet, dietary beliefs and various dietary errors. 
It is also appreciated the patients' adherence to the various advice of their doctors. In addition, this study focuses on monitoring and surveillance with specific indicators and the cardiovascular risk incurred by these patients. Our study also addressed:

a) The individualisation of the care of diabetics

b) The involvement and adherence of diabetics in this medical care, to establish an updated health status of diabetic patients

\section{Materials and Methods}

This survey is descriptive. It aims to describe the knowledge of patients with type 2 diabetes with regard to the rules of diet and diet. This is a quantitative, prospective study belonging to the group of descriptive cross-sectional observational studies. The survey took place over a semester from October 2017 to March 2018. For this, we developed a questionnaire of 73 items [1,2].

\section{Study Population}

We chose to study the diabetic population from three municipalities (El Marssa, Sidi Abderrahmane and El Guelta) who consults in the health center of Sidi Abderrahamne, the polyclinic of El Marssa and the center of El Guelta.

a. Target Population: In 2017, according to the figures collected near the municipalities, there are 8000 inhabitants in the municipality of Sidi Abderrahmane, 14000 in El Marssa and 4000 in El Guelta.

The prevalence rate of diabetes in 2012 was 17, 3\%, of which $57 \%$ were men and $43 \%$ women and an average age of 68 years.

b. Inclusion Criteria: Type 2 diabetes

c. Exclusion criteria: Chronic diseases other than diabetes

d. Sampling: We printed 100 questionnaires. Our minimal goal was a return of 50 responses and the optimal goal of 100 responses.

\section{How to Recruit Patients}

The consultation took place in each health center ( 3 days of the week), from $8 \mathrm{~h} 30$ to $14 \mathrm{~h}$ or $16 \mathrm{~h}$. It includes an interrogation (questionnaire), a clinical examination, anthropometric measurements and bio-impedancemetry. The duration of the consultation may exceed 30 minutes, a second meeting is necessary to review the balance sheets and especially the glycemic selfmonitoring of 3 days before the next consultation. The second consultation also includes providing nutrition advice to patients.

\section{Collection of Data}

The filling of the questionnaire is done during the initial consultation, then the analysis (according to the food survey and the medical examination), will be carried out during the second consultation.

\section{Questionnary}

The questionnary breaks down into nine parts, with a total of 76 questions. The majority of questions are closed-ended (with several possible answers).

First Part "Identification": This section collects the information defining the population of our study: sex, age, residence, sociooccupational category, age of diabetes, personal and family history, associated pathologies, biological assessment (glycemia, HbA1c, lipids, uric acid, creatinemia).

Second Part «Education: Have you been informed about? Here, the main data to be determined is the existence of information delivered to the patient during his previous management: selfmonitoring, complications, treatments; the patient is asked about his satisfaction with the information received.

Third Part "Knowledge about the Disease": The objective is to evaluate the knowledge on the pathology, the risk factors, the evolution of the diabetes and the measures hygiene and dietary.

Part Four "Knowledge and Beliefs about Diabetic Nutrition": This part seeks to answer to appreciate the state of knowledge of the patients with regard to the food, the modes of food consumption and the beliefs towards certain foods, the interest of the food and finally the observance of a diet or no.

Part Five: "Eating Habits": This section attempts to evaluate daily eating habits: eating habits, frequency of meals, snacking, oils used, and changing or not eating habits.

Part Six "Usually, do you Eat?": In this part, the objective is to evaluate the frequency of daily, weekly or monthly food intake (during the month), for the different food categories: cereals, dairy products, fruits vegetables, meats, sweets, drinks, used fat, adding salt and some traditional meals.

Part 7 "What Kind of Physical Activity do you Practice?": This part evaluates the nature and intensity of the patient's physical activity.

Part Eight "Cardiovascular Risk Factors": The cardiovascular risk of the patient is estimated by including other risk factors: stress, smoking (passive) and quality of sleep (insomnia, existence or not of sleep apnea syndrome).

Part 9 "Follow-Up Indicators": This last part seeks to evaluate the quality of patient monitoring.

\section{Calculation Tools and Statistical Studies}

The study data were entered using computer calculation software (SPSS 14.0). The descriptive analyzes of the tables were carried out with the same software. Descriptive analysis included means and standard deviations for qualitative data and percentages for data. 


\section{Results}

Figures (1-5)
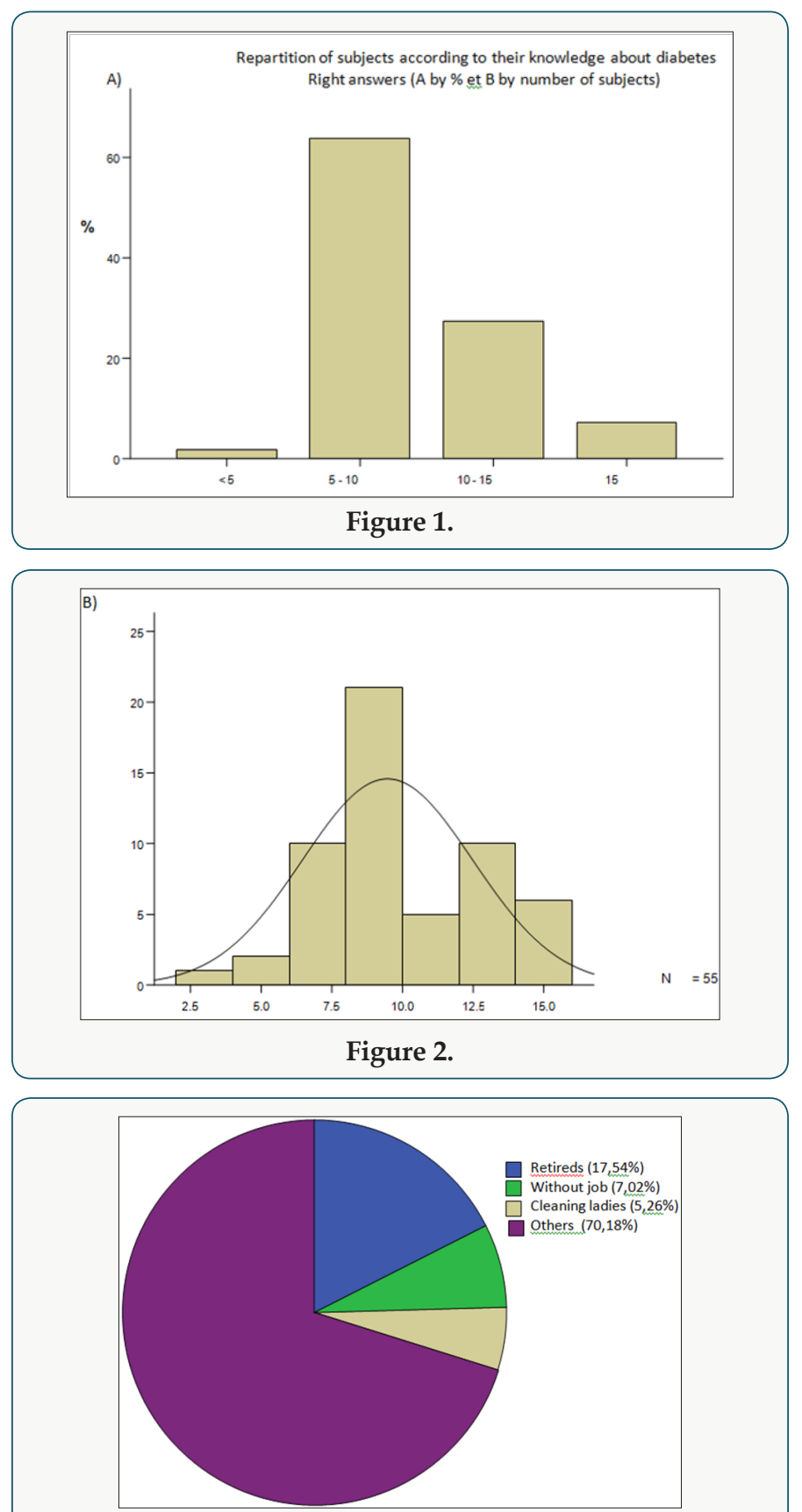

Figure 3: Repartition of patients according to their activities

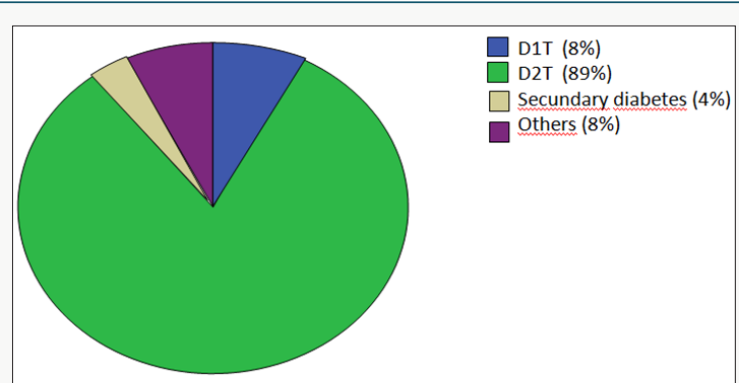

Figure 4: Repartition according to the type of diabetes.

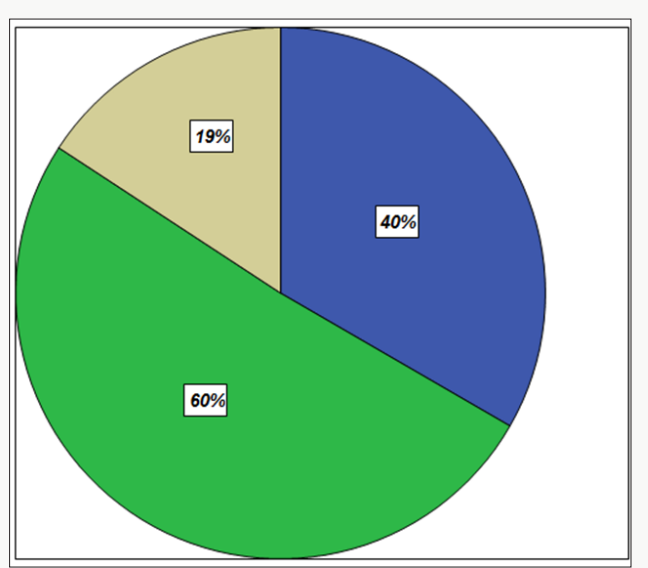

Figure 5: Rate of follow-up of patients by a specialist: $40 \%$ of subjects are followed regularly by a specialist while the majority never consults with a specialist (60\%).

\section{Discussion}

All the recommendations recognize that dietary and hygiene measures remain one of the fundamental bases of the management of diabetic states, whether of type 1 or 2 . However, it is generally advisable to align the drug treatments and dietary and lifestyle measures (DLMs) of diabetic especially type 2, throughout its evolution, even if the effectiveness of nutritional intervention trials in this area is always more difficult to prove than that pharmacological studies. The arguments in favor of DLMs are supported by the following arguments:

a. In primary prevention, DLMs are generally more effective than pharmacological interventions in preventing the conversion of a borderline condition, glucose intolerance into patent type 2 diabetes

b. It was also found that diabetics who do not respect the minimum of these DLMs are rarely well balanced hence the need for earlier drug escalation (mono and dual therapy, then triple therapy, or insulin therapy

c. In addition, dietary measures must be implemented or reinforced in diabetics insulins under penalty of additional weight gain and abuse (it is commonly accepted that a $1 \%$ average fall in HbA1c insulin treatment in a type 2 diabetic causes an average weight gain of the order of $2 \mathrm{~kg}$ ); DLMs have priority over anti-obesity drugs that lead to several side effects.

All these observations argue in favor of nutritional measures which must be considered as an unavoidable step in the overall care of the diabetic. However in reality, things are not so simple: nutrition as a discipline does not always occupy an important place in the medical act; the influx of medicines sometimes makes us lose the sense of the usefulness of an adapted nutritional care; Finally, nutrition is still often controversial, including diabetes, which causes confusion and inaction (because nutritional intervention studies are difficult to prove). 
In 1985, DLMs reached a new stage: Americans discovered the beneficial antiatherogenic and anti-thrombogenic properties of monounsaturated fats (MUFAs). It was at this time that we began to hunt for lipids, particularly saturated fatty acids, accused of all ills and favor MUFA. From this date appeared several recommendations, many clinical trials concerning these RHD and several regimes have been developed among them the Mediterranean diet that has continued to evolve over time but is still fashionable for the prescriber [3-6]. Thus, it appears that nutrition is an area where these recommendations are subject to permanent reassessment. The dietetics of diabetes mellitus is no exception to this rule. The nutritional means to achieve these objectives are four in number:

a) Act on the amount of carbohydrates ingested at each meal

b) Act on the nature of carbohydrates

c) Increase the fiber content of foods

d) Associate other nutrients with dietary carbohydrates

\section{In Interprandial Period}

Avoid excessive glycemic lowering and especially hypoglycaemic episodes at a distance from meals. This precaution is more theoretical than practical in type 2 diabetics treated with oral antidiabetic drugs, although some diabetics treated with insulin secretagogues (sulfonylureas or glinides) may experience hypoglycaemia at a distance from meals, in the late morning, but more readily at the end of the morning. In our study, it is clear that the socio-economic level, the school level and the difficulties of access to specialized care are largely responsible for the failures of an adapted management of the diabetes management, in particular in as regards the hygiene aspect of life (marked by a generalized sedentary lifestyle) and the nonobservance of dietary rules due to erroneous beliefs about diet.

\section{Conclusion}

The main goal of nutritional management is to promote in diabetics a better lifestyle change (eating habits, physical activity, relaxation, leisure) to improve the metabolic control of the disease and its evolution.The goals of nutrition in diabetic patients are twofold: a. Actions on glycemic disorders: Normalize blood glucose and avoid or minimize extreme blood sugar fluctuations in the direction of hyper or hypoglycemia (acute hyperglycemic peaks that usually occur after meals and excessive blood sugar drop that can lead to hypoglycaemic episodes more or less severe at a distance from meals).

b. Actions to fight the risk factors frequently associated with diabetes (dyslipidemia, high blood pressure, hemostasis disorders) and which make the bed of cardiovascular complications.

In fact, HDMs are not dedicated to regulating blood glucose alone (which is a restricted or glucocentric management) but they are part of a broader vision in a comprehensive management of the diabetic and as the experts said (it does not treat not a diabetes but we treat a diabetic).These objectives must respect the multiple dimensions of lifestyle, be they cultural, social, family and/or religious. For this reason the prescription must be personalized and negotiated (discussed with the patient and if possible with those around him) taking into account the particularities of each diabetic and his financial possibilities in particular. MHD placement should be preceded by the assessment of the starting dietary profile with the prescriber.

\section{References}

1. American Diabetes Association (2015) Classification and Diagnosis of Diabetes Care 38(Suppl S8-S16).

2. Eberhart MS, Ogden C, Engelgau M, Cadwell B, Hedley A (2004) Prevalence of Overweight and Obesity Among Adults with Diagnosed Diabetes United States, 1988-1994 and 1999-2002. Morbidity and Mortality Weekly Report 53(45): 1066-1068.

3. Eves ND, Plotnikoff RC (2006) Resistance training and type 2 diabetes Considerations for implementation at the population level, Diabetes Care 29(8): 1933-1941.

4. Knowler WC, Barrett Connor E, Fowler SE, Hamman RF, Lachin JM (2002) Reduction in the incidence of type 2 diabetes with lifestyle intervention or metformin. N Engl J Med 346: 393-403.

5. Lagger G, Pataky Z, Golay A (2010) Efficacy of therapeutic patient education in chronic diseases and obesity. Patient Educ Couns 79(3): 283-286.

6. WHO (2003) Diet, Nutrition and Chronic Disease Prevention - Technical Report Series 916. World Health Organization, Geneva, p. 128, chapter 5.3 (“Recommendations for Diabetes Prevention") pp. 62-65.
This work is licensed under Creative Commons Attribution 4.0 License

To Submit Your Article Click Here: Submit Article

DOI: $10.32474 /$ CTGH.2018.01.000102

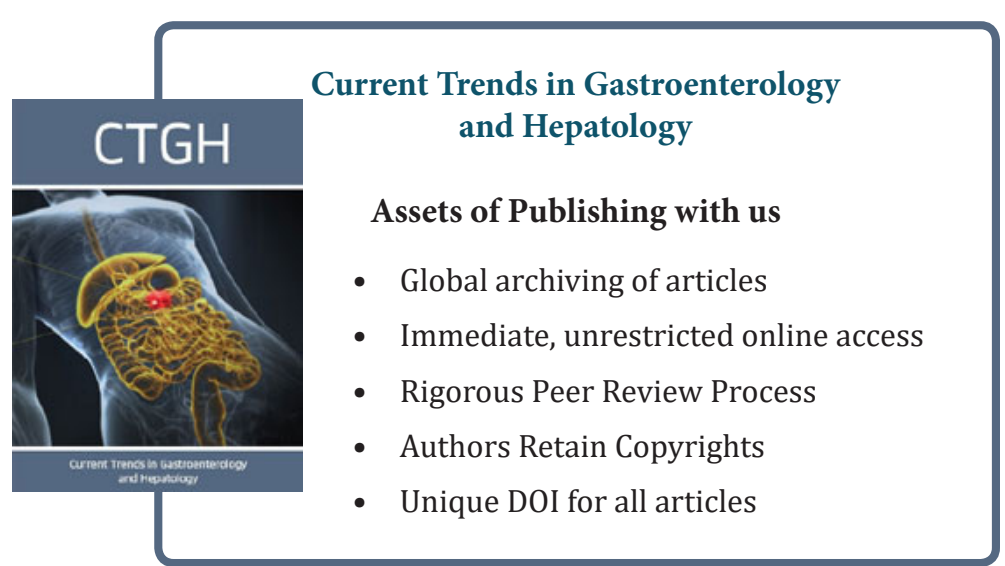

